Acc。 213821

Station Record

SMITHSONIAN INSTITUTION-BREDIN EXPEDITION

to

The Society and Tuamotu Islands, April \& May, 1957

Scientist participating

Waldo I. Schmitt, Leader

Harald A。 Rehder

Thomas E。 Bowman

Charles E。 Cutress

No. 1 - ApriI 4. Eighty yards E. by E.S.E。 of Motu Uta, Papeete Harbor, Tahiti. Depth $3 \mathrm{ft}$. Sandy bottom with scattered coral.

No. 2 - April 8, 2:45 P.M. Papeete Harbor, Tahiti. Plankton tow. Foot net, No. 12 bolting silk, 8-10 ft. below surface.

No. 3 - April 8, 2:55 P.M. Papeete Harbor, Tahiti。 From anchor chain of yacht "Mareva". Depth of anchor estimated to be 11 meters. Anchor had been down about 4 months. No specimens from bottom portion of chain.

No. 4 - April 9, 6:30-6:45 A.M。 About 20 miles N.N.E. of Papeete, Tahiti. Plankton tow. Foot net, No. 12 silik, all line out, $50^{\circ}$ wire angle, ship moving very slow。

No. 5 - April 9, 7:15-8:00 A.M。 Same position and equipment as No. 4. Some of the copepods bright blue.

No. 6 - April 9, 9:13-9:56 A.M. Iocality about the same as No. 5 but slightIy closer to Papeete. Equipment same as No. 5o. Sunny, clear, tow made just below surface.

No. 7 - April 9, 2:10-2:22 P.M. About 5 miles N. of Tahiti。 Other data same as No. 6.

No o 8 - April 1.0, 10-12 A.M。 Reef No of Motu Uta Island, Papeete Harbor, Tahiti.

No. 9 - April 10, 2:50 P.M. Quai Bir Hachem, Papeete Harbor, Tahiti. From anchor chain of Yacht "Mareva".

No.1.0 - April 11, 4:30-5:30 P.M. Tikahau, Tuamotu Islands。 Patch reef in lagoon, 300 yds, from deep water pass.

No.IOa- Same as No. 10. Specimens removed from coral.

No.11 - April 12, 10-12 A.M。 Tikahua, Thamotu Islands. South of deep water pass. Terrestrial, under brush and rocks in coconut grove. 
No。 12 - April 12, 10-12 A.M。 Tikahau, Tuamotu Islands, Matiti Island south of deep water pass, along lagoon shore.

No. 13 - April 12, 10-12 A.M. Tikahau, Thamotu Islands, Matiti Island, ocean reef.

No. 14 - April 12, 10-12 A.M. Tikahar, Tramotu Islands, Causeway S. of Matiti Island, Depth 0w2 fto

No. $14 a$ - Same as No. 14 but on lagoon side of causeway。

No. 15 - Aprol 12, 3:30-5:15 P.M. Tikahau, Thamotu Islands. Matiti Island, 10-20 ft. inland from lagoon skore.

No. 16 - ApriI 12, 3:30-5:15 P.M. Tikahav, Thamotu Islands。 Matiti Island, 20 yds. inland from lagoon shore, rear concrete cistern.

No. 17 - Aprol 12, 3:30-5:15 PoM。 Tikahau, Tuamotu Islands。 Same locality as No. 14, near shore.

No. 18 - AproI 12, 4:00 P.M. Tikahau, Thamotu Islands, South of deep water pass, patch reef in lagoon, at "Mareva" anchorage. Depth 50 ft.

No. 19 - April 12, 8:15 P.M. Tikahau, Thamotu Islands. Patch reef in lagoon S。 of deep water pass. Plankton tow from rowed skiff, in vicinity of "Mareva" anchorage. Depth of: net 10 $15 \mathrm{ft}$.

No. 20 - Aproil 13, 7:30-9:00 AoMo Tikakau, Tuamotu Islands. North of deep water pass, 300 yds. off fiaking villiage. Depth 20-30 ft. Rectangular and triangular dredges plus aqualung.

No. 21 - Apr:II 13, 11:30-11:40 A。M。 Tikahau, Tuamotu Islands。 Just WoN.W. of Mai.ia.i. Island。 Plankton tow, foot net, depth surface to $12 \mathrm{ft}$.

No. 22 - Aproi 13, 1:30-3:30 PoM。 Tikahaiu, Thamotu Islands。 Maiai Island, outer side, just above tide level of old coral rampart.

No. 22a- Same as No. 22 except collection from base of ccral rampart.

No. 23 - Aproil I3, 1:30-3:30 PoM。 Tikahau, Thamctu Islands, Maiai Island, just shcreward of outer edge of ocean reef.

No. 24 - Aprol 13. Tikałar, Thamotu Islands. Ma:ai Islara, ocean reef, along old coral rampart.

No. 25 - April 13. Tikahan, Tuamotu Islands, Maiai Island, interior, leaf mold under Pisonia trees.

No. 26 - Apr:1 13, 3:30-5:00 P.M。 Tikahau, Thamotu Islands, Maiai Tsland, lagoon sbore.

No. 26a- Same as No. 26 except specimens from brackish pond. 
No. 26b-Same as No. 26 except -specimens from cove。

No. 26c- Same as No. 26b。

No. 27 - April 14. Tikahau, Tuamotu Islands. Maiai Island, lagoon shore, cove。 Seine。

No. 28 - April 14. Tikahau, Tuamotu Islands. Maiai Island, lagoon, patch reef near shore.

No. 29 - April 14. Tikahau, Tuamotu Islands, Maiai Island, ocean reef, outer edge.

No. 29a- Same as No. 29 except specimens taken near coral rampart.

No. 30 - April 14. Tikabau, Tuamotu Islands. Maiai Island, lagoon shore.

No. 31 - April 14. Tikahau, Tuamotu Islands. Maiai Island, brackish pond. 50 fit. from lagoon shore.

No. 3la- Same as No. 31 except specimens taken on shore between brackish pond and No. 27 .

No. 32 - ApriI 14。 Tikahau, Tuamotu Islands, Off Maiai Island, dredging, depth 5 fathoms, trapezoidal dredge.

No. 33 - April 14, 3:30-5:00 P。M。 Tikahau, Tuamotu Islands. North shore of Matiti Island.

No. 34 - ApriI 14, 9:30-11:00 PoM。 Tikahau, Tuamotu Islands. Matiti Island, part ocean shore; part inland on lagoon side.

No. 35 - April 15. Tikahau, Tuamotu Islands. Patch reef off Matiti Island. Rotenone station.

No. 36 - ApriI 15. Tikahau, Tuamotu Islands。 Causeway S。 end of Matiti Island, near shore.

No. 37 - April 16. Makatea, Tuamotu Islands。 Temao Harbor, north of pier.

No. 38 - Same as No. 37 except (soil samples) from beneath cliffs behind reef.

No. 39 - April 17, 9:30-10:00 P.M。 Papeete Harbor, Tahiti。 Night light over side of "Mareva" at landing, dip and tow net.

No. 40 - April 19. Papeete Harbor, Tahiti. Reef N。Wo of Motu Uta Island。 Rotenone station.

No. 41 - Same as No. 40 except collection donated by a native.

No. 42 - April 20. Papeete Harbor, Tahiti. Outer reef N.W. of Motu Uta Island. 
No. 43 - April 20. Papeete Harbor, Tahiti, "Mareva" anchorage. Night light。 dip net。

No. 44 - April 18-19. Papeete, Tahiti. Fish market。

No. 45 - April 21. Papeete, Tahiti。 Fish market。

No. 46 - April 21. Punaauia District, Tahiti. Reef N. of Taapuna Pass, depth $1-31 / 2 \mathrm{ft}$.

No. 47 - April 22. Fanui Bay, Bora Bora Island. South side of bay and E。 of landing near pass.

No. 48 - April 22. Bora Bora Island. South of Farepiti Point.

No. 49 - April 22. Bora Bora Island. Landing E。 of Farepiti Point. Night light, dip net。

No. 50 - April 23. Bora Bora Island. Farepiti Point landing. Purchased.

No. 51 - April 23. Bora Bora Island. 3/8 mi.S。 of Farepiti Point, fringing reef。

No. 5la- Same as No. 51 except near shore. Sorted from broken pieces of coral.

No. 52 - April 24, 8-8:39 P.M。 Bora Bora, landing E。 of Farepiti Point. Dip net and light. Ostracods. With bright bluish-green luminescence. Many hyperiid amphipods.

No. 53 - April 22。 Bora Bora, landing E. of Farepiti Point. Ligia collected along dock.

No. 54 - April 22. Borra Bora; 150 yds. E. of Farepiti Point landing. Under leaves on damp ground near water faucet.

No. 55 - April 25, 9:10-11:00 A.M. Bora Bora, off Tereia Point。 Plankton, foot net, well below surface, bottom, approximately 15 fathoms. Five tows combined.

No. 56 - April 25. Bora Bora, N. of Tereia Point. About 200 yds off shore。 depth 13-16 fathoms, shell bottom. Trapezoid dredge.

No. 57 - April 25. Bora Bora, N. side Fanui Bay, from Tereia Point to old navy dock. Dredging, about 150 yds。 off shore, depth 13-14 fathoms, fine snad and coral fragments. Trapezoid dredge.

No. 58 - April 25. Bora Bora, Tereia Point. Coral reef and sand bottom, near shore leeward side。 Depth 2-4 ft.

No. 59 - April 24. Bora Bora, Tevairoa Island. Purchased from fisherman Pinna and Grapsus.

No. 60 - April 25. Bora Bora, Motu Ahuna N. of Tearvauni Pass。 Two species of Cypraea from reef purchased from boy. 
No. 61 - April 25. Bora Bora, Vaitape village. Mollusk from pieces of coral along wharf.

No.62 - April 25. Bora Bora, Wo of Motu Tapu. Lagoon side of ocean reef.

No. 63 - April 25, 8:30-9:00 P.M。 Bora Bora, mooring $W_{\circ}$ of $N$ 。 end of Toopua Island. Dip net and light, bottom about 7 fathoms. Mostly crab larvae.

No. 64 - April 26. Bora Bora, S.E。 side of Motu Tapu Island。 Ghost and hermit crabs from corals and beach; millipede under debris above high tide; other crabs from dead coral along N.W. shore。

No. 65 - April 26. Bora Bora, Motu Tapu Island. Melania numerous in F.W. pond in middle of island.

No. 66 - April 26. Bora Bora, Wo of Motu Tapu. Transect of leeward barrier reef. Six collections made in all, at 15-20 ft。 intervals, starting with No. I on ocean side and ending with No。 6 at edge of moat.

No. 67 - April 26. Bora Bora. Three specimens from local reefs. Collected and donated by M. Henri Moua, of Vaitape.

No. 68 - April 26. Bora Bora. Vaitape pier. Dip net and flashlight. Stenopus, Parthenope, Ligia, and Stichopus from sides of pier.

No. 69 - April 27. Bora Bora, Muller Reef, S. of Teavanui Pass. Rotenone station lagoon side of ocean reef。

No. 70 - April 26, 8-10P.M. Bora Bora, Wo of Toopua Island。 Outer ocean reef。 Lobsters and crabs taken by lantern and flashlight.

No. 71 - April 27. Bora Bora, E. of landing at Farepiti Point. One Calappa taken in sand and 6 in. water.

No. 72 - April 27, 5-6:00 P.M。 Bora Bora, E. of landing at Farepiti Point. From dead coral boulders.

No. 73 - April 28. Uturoa, Raiatea. Damp ground along open sewer. Talitrid amphipods, tupa, fiddler crabs, and Lygia.

No. 74 - April 28, 4:00 P.M。 Uturoa, Raiatea. Sand flat E。 of protestant church。 Depth 1-3 ft.

No. 75 - April 28, 2:30-4:30 P.M. E. of dock at Uturoa, Raiatea. From coral boulders in muddy water. Shrimp, palaemonids, alpheids, and trapezius.

No. 76 - April 28. Uturoa, Raiatea, Riviere Vaipao. F. W. station, above cement block factory. Melanoides on rocks, uncommon.

No. 77 - April 28, 9-11:00 P.M. Same locality as No. 74。 Coleman lantern. Holothurians, some with Lissocarcinus. 
No. 77 a - Same station as No. 77. Collection made from 6-7 in. piece of coral.

No. 78 - April 29. Raiatea, Uturoa, Taoru Island. Terrestrial station. Isopods, snails, termites, centipedes, millipedes, scorpions and moss.

No. 79 - April 29. Raiatea, Uturoa, Taoru Island. Reef near shore, shallow water.

No. 80 - April 29. Raiatea, Uturoa, N. end Taoru Island. Branching coral, depth 2-3 ft.

No. 80 a - Same station as No. 80. Collection from pieces of coral.

No. 81 - April 29. Raiatea, Uturoa, S.W. end Tetaro Island. Reef near shore, depth 2 ft.

No. 81a - Same station as No. 81. Collection from pieces of coral.

No. 82 - April 29, 7:30-8:15 P.M. Raiatea, Uturoa, W. of Taoru Island. Dip net and light. Mostly fish and crab larvae.

Ho. 83 - April 30, 10:45-10:55 A.M. Raiatea, Point Faatiaapiti, Opoa. Plankton tow, foot net, bright sunshine.

No. 84 - April 30. Huahine, Baie de Maroe. Sandy shoal, depth 2-3 ft. Limnoria from wooden post.

No. 84a - Same station as No. 84. Collection from pieces of branching coral.

No. 85 - April 30, 7:30-9:00 P.M. Huahine, Port du Bourayne. Dip net and light. Luminescent ostracods and one shrimp.

No. 86 - May 1. Huahine, Port du Bourayne. Sandy reef.

No. 86a - Same station as No. 86. From Halimeda.

No. $86 b$ - Same as No。 86. From branching coral.

No. $86 \mathrm{c}$ - Same as No. 86. From massive coral.

No. $86 \mathrm{~d}$ - Same as No. 86c. Dark brown sponge crab.

No. 87 - May 1. Huahine, Port du Bourayne. Shrimp and oysters purchased from fisherman.

No. 88 - May 1. Huahine, Port du Bourayne, S. Wo of Vaiorea Island. From reef and several pieces of eroded coral.

No. 89 - May 1, 7:45-9:00 P.M。 Huahine, Baie Haamene, I/2 mi. S。 of Fare. Dip net and light. One young flying gurnard. 
No. 90 - May 2. Huahine, Point Teffaar No of Baie Fare and S。 to Point Teopape. Coral reef, depth 0-4 ft. Includes 4 talitrids from sandy beach.

No. 90a - Same station as No.90. Collection from pieces of dead coral.

No. 91. - May 2. Huahine, Baie Fare, 100 yds from Point Teopape. F. W. pond, green with algae, Melanoides.

No. 92 - May 2. Huahine, Baie Fare, S. of Passe Avamoa. Sand pockets in reef, depth 4 ft., 2 species of Conus.

No. 93 - May 2. No end Huahine, Lac Maheva. Bivalves purchased at Fare.

No. 94 - May 2, 4-5:00 P.M. Huahine, No of Baie Fare. From piece of branching coral. (Preservative not added - collection lost.)

No. 95 - May 3. Huahine, S。 of Passe Avamoa, near Fare。 Rotenone station and general collecting on outer reef.

No. 96 - May 3. Huahine, Baie Haamene. Small fish netted at surface, depth 12 fathoms.

No. 97 - May 3. Huahine, Lac Maheva, Maheva village. F. W. station. Crabs, mollusks, and a fish.

No. 98 - May 3. Huahine, Fare. Turbo from waters edge on concrete quay.

No. 99 - May 4, 9-9:30 P.M. Tahiti, Papeete Harbor. Dip net at "Mareva" anchorage.

No. 100 - May 6, 8-10:00 P.M. Moorea, Opunohu Bay. (=Papetoai Bay), Kellum's landing. Dip net and light. Hyperiid amphipods and herring, the latter numerous.

No. 101 - May 6-7. Same locality as No.100. Overnight crab trap baited with Puss and Boots cat food. Depth 10-12 fathoms. One crab, one goatfish.

No. 102 - May 7. Moorea, S.E。 end of Opunohu Bay。 Sandy beach. Talitrid amphipods and oniscid isopods.

No. 1,03 - May 7, 1-2 P.M. Moorea, Toatane Reef $W$ of entrance to Paopao Bay。 Collection from reef.

No. 104 - May 7. Moorea, Paraoro Village. Damp soil under vegetation in vanilla field behind village. Sow bugs, millipedes etc。

No. 105 - May 8, 7:30-10:00 A.M。 N.W. Moorea, Wo of Point Hauru. Collection from reef。

No. 106 - May 8. N.W. Moorea, N.W. Motu Fareone Island. Fossil coral along shore. No. 107 - May 8. Moorea, Opunohu Bay, Darr's landing。 On rocks along shore. 
No. 108 - May 8. Moorea, Wo fork of Opunohu River. F.W. station. Shrimp, snails and fish.

No. 109 - May 9. Moorea, E. side of entrance to Opunohu Bay. Bottom sand and coral, depth 2-16 ft.

No. 109a- Same station as No. 109. From piece of branching coral.

No. 109b- Same as No. 109a. From base.

No. 110 - May 9. Moorea, Opunohu Bay, Banc Touria E. side of Passe Taareu.

No. 111 - May 9. Moorea, Opunohu Bay. Rocks at S.W. corner of bay. Neritina, Iittorina and dead Asaphis from black sand beach nearby。

No. 112 - May 9, early afternoon. Moorea, Opunohu Bay, F. s1de of entrance. dredging, depth 27-29 meters, bottom Halimeda.

No. 112a- Southern end of station No. 112. Bottom stony, barren.

No. 113 - May 9. Moorea, Paopao Bay, K. of Passe Avaroa. Mollusk trap in moat behind reef.

No. 114 - Same station as No. 112. Several plankton hauls combined, foot net.

No. 115 - May 10, Moorea, Opunohu Bay, Kellum's landing. Littorina on pilings.

No. 116 - May 10. Moorea, Maharepa Bay, W. of Irihonu Pass.

No. 116a- Same station as No. 116. Collection from pieces of coral pavement.

No. 117 - May 10, Moorea, Maharepa. Helcioniscus from exposed concrete beacon near wharf.

No. 118 - May 10, Moorea, Maharepa landing. Littorina from concrete pier.

No. 119 - May 10. Moorea, Maharepa. Sandy beach adjacent to landing. Flashlight. Talitrid amphipods on shore.

No. 120 - May 11. Moorea, Maharepa. Sandy beach adjacent to landing, depth I ft.

No. 121 - May 11. Moorea, Maharepa. Sandy bottom off landing, mollusk traps, depth 10-25 ft.

No. 122 - May 11. Moorea, S.W. shore of Lake Temae. F.W. station, amphipods from reeds in water. Isopods from damp shore.

No. 123 - May 11. Moorea, S.W. shore of Lake Temae. Talitrid amphipods from under debris in taro field.

No. 124 - May 11. Moorea, small stream flowing into Lake Temae. Melanoides on rock.

No. 125 - May 11. Moorea, NoE. shore of Lac Tamae. Melanoldes from stones and debris near shore. 
No. 126 - May 11, 3:30-5:00 P.M. Moorea, Nuarei Bay。 Reef opposite Point Teanatira.

No. 127 - May 11。 10:00 A.M. - 12:30 P.M. Moorea, Nuarei Bay. Cracked from pieces of coral on shallow sandy shore.

No. 127a- Same station as No。127. Olives, cones, Culcita and Acanthaster from under pieces of coral.

No. 128 - May 11。 Moorea, W. side Nuarei Bay。 Electric light, sandy shore 1/2$21 / 2 \mathrm{ft}$.

No. 129 - May 12. Moorea, Nuarei Bay, ocean reef. Rotenone station.

\section{Miscellaneous Collections}

M-I Jan. 1957. N.W. Moorea。 On coral head on sandy bottom, depth I ft. Two galatheids. Coll. \& don., J.E. Randall.

M-2 April 5, 1957. Murex ramosus bought in Papeete, Tahiti by J. E. Randall. Said to have come from Bora Bora.

M-3 April 7, 1957. Scylla bought in Papeete, Tahiti, by J. E. Randall.

M-4 One cone collected at Paea, Tahiti, near $\mathrm{Km}$.26, reef opposite March's place. Donated by Dr. H. N. March。

M-5 May 9, 1957. Three mollusks from Motu Fareone, Moorea. Coll. \& don., by $M_{0}$ Kellum.

M-6 Moorea. Several mollusks coll. \& don by Medford Kellum. Two Cypraea Iynx are from Kellum place, Paea, Tahiti, near Km. 26. 
Check spelling

Sen

Smithsonian Institution-Bredin Expedition to

The Society and Tuamotu Islands, April \& May, 1957

Sta. 18-57 Apr. 12, 4:00 Pom. Tikinau, Tuamotu Islands. South of deep water pass, patch reef in lagoon, at "Mareva" anchorage. Dept 50 st.

Sta. 26-57 April 13,3:30-5:00 Pam. Tikihau, Tuamotu Islands. Maia Island, lagoon shore.

Sta. 37-57 Mefetea, Tuamotu Islands. Temao Harbor, north of pier.

Sta. 62-57 April 25. Bora Bora, W. of Mot Tape. Lagoon side of ocean reef.

Sta. 90-57 May 2. Huhine, Point Teffar N. of Bail Fare and S. to Point Teopape. Coral reef, depth 0-4 It. Includes 4 talitrids from sandy beach.

Sta. 95-57 May 3. Huahine, S. of Passe Avamoa, near pare. Rotenone station and general collecting on outer reef.

Sta. 116-57 May 10. Moored, Maharepa Bay, W. of Irihonu Pass.

Sta. 103-57 May 7, 1-2 pom. Moored, Toatane Reel W. of entrance to Papa Bay. Collection Exon reef.

Sta. 105-57 May 8,7:30-10:00 a.m. N.W. Korea, W. of point nauru. Collection from reef. 